\title{
reviews
}

IN the diversity of today's astrophysics, where does radio astronomy stand? That question arises when reading the book by the staff of the National Radio Astronomy Observatory (NRAO) in the United States, a book whose title aims without restriction at the entire Universe beyond the Solar System. One would expect it, then, to cover the vast terrain of astrophysical interactions and phenomena, as seen in the radio window's perspective. A survey of the approximately 1,000 entries in the subject index confirms that indeed an immense variety of objects and processes are touched upon. There are 12 chapters each by a single author; the only chapter on technique per se, on "Interferometry and Aperture Synthesis", was written by a pair of authors, synthesising expertise nurtured at Caltech and Cambridge, respectively. It is a wise choice of the editors and a valuable contribution by these authors, providing so clear an exposition of what is the outstanding observation technique at radio wavelengths whenever imaging elements smaller than one arc minute, positional accuracy better than one arc second, and sensitivities per beam lower than a few milli Jansky are called for. Having begun using $1-5-\mathrm{km}$ arrays so successfully at decimetre and centimetre wavelengths, this extremely versatile technique that so thoroughly exploits current computer capacities, is spreading to millimetrewavelength astronomy and to transcontinental interferometry. The chapter on observation (and reduction) technique conveniently separates the first nine

\section{Bridging the gap in radio astronomy}

\section{H. van der Laan}

Galactic and Extra-Galactic Radio Astronomy. By the Staff of the National Radio Astronomy Observatory. Edited by G. L. Verschuur and K. I. Kellermann. Pp.x +402. (Springer: Berlin and New York, 1974.) DM 98.30; $\$ 40.10$.

chapters that deal with galactic subjects, from the last three, which explore extragalactic objects.

The book more than spans the gap that exists between current textbooks and current reviews in radioastronomy. (Of the former there are, because of the swift developments in all fields of astronomy, only one or two worth buying; the latter are, however, numerous, both under auspices of the International Astronomical Union and in several annual and summer school series.) There is no editorial uniformity in the treatment of first principles or the completeness with which current insights are presented. The editors seem to have limited their rule to the choice and sequence of chapters and authors, and thus achieve coherence without blurring their authors' diversity. Some of the chapters are authoritative reviews, others unpretentious sketches, and a few are highly personal accounts. All are worth reading, some deserve and require close study. Many chapters, singly or in groups, provide an admirable means of progressing soundly from basic matters to current journal literature; thus they attain a major purpose of all postgraduate courses. Active research astronomers found time to write this excellent book and aspiring researchers will spend their time well in reading from it, to each his own selection.

No completeness is possible on this vast plain of astronomy. Of the omissions only the topic of continuum emission from normal galaxies and their nuclei is regrettable, all the more so since the present director of this same NRAO did the pioneering work. But I regard this book as a fitting closure of radioastronomy's adolescence. Well nigh every astronomical object and astrophysical process is now touched by this technique and this scope makes it imperative to deal with themes and subjects in a multispectral context; this is the case even when discussing objects that are first and foremost radio sources.

Symposia or books confined to radio astronomy will increasingly combine subjects that lack all affinity and separate those which are naturally all of one piece. To acknowledge this means to adapt courses of instruction, conferences and even the structure of organisations such as the International Astronomical Union; it is the recognition that radioastronomy has come of age.
Now that Magnus Pyke has become a television personality, known to the public for his extraordinary antics which somehow succeed in explaining scientific principles, a new book bearing his name might be expected to use the same type of technique. Those who buy Success in Nutrition expecting a good laugh combined with a little painless and easily assimilated knowledge are likely to be disappointed. This book, although well-written and easy to read, is a straightforward and orthodox textbook, aimed at ' $O$ ' level and ' $A$ ' level students of food and nutrition, ' $A$ ' level students in home economics and those taking OND and HND courses in catering and hotel management. It is also recommended for the serious reader following a self-study course. As a

\section{Pyke's guide to nutrition}

Success in Nutrition. By Magnus Pyke. Pp. $x+227$. (John Murray: London, 1975.) £1.95.

textbook it is excellent. All the various constituents of our diet are described, details being given of their occurrence in different foods, and the effects of cooking and preparing them on the different ingredients. Dr Pyke is always careful to point out gaps in our knowledge, and to ridicule the absurd statements produced by the faddist and the crank. Some readers may think that he is overgenerous in his commendation of the food-processing industry. The only gap in the text is the omission of any statement about 'roughage'. There seems little doubt that civilised people may suffer from the lack of fibre in their food, and that its replacement relieves constipation and many more serious complaints. I would have been interested in Dr Pyke's comments. Kenneth Mellanby 